\title{
CTRP Biomarker Terminology
}

National Cancer Institute

\section{Source}

National Cancer Institute. CTRP Biomarker Terminology. NCI Thesaurus. Code C142799.

A set of terms that is comprised of the biomarkers referenced in $\mathrm{NCl}$-sponsored clinical trials. 